\title{
Réponses analytiques aux syndromes cholinergiques et anticholinergiques
}

\section{Cholinergic and anticholinergic syndromes : laboratory testing}

\section{Jean-Pierre GOULLÉ*(1), Jean-Michel DROY( ${ }^{(2)}$, Jean-Philippe LEROY ${ }^{(2)}$}

(1) Laboratoire de Pharmacocinétique et de Toxicologie Cliniques, Groupe Hospitalier BP 24 - 76083 LE HAVRE Cedex - Tél : 33232733223 - Fax : 33232733238

(2) Centre de Toxicologie Clinique et de Toxicovigilance, Hôpital Charles Nicolle, 1, rue de Germont 76031 ROUEN Cedex - Tél : 33235884400 - Fax : 33232888128

*Auteur à qui adresser la correspondance : Jean-Pierre GOULLÉ, Laboratoire de Pharmacocinétique et de Toxicologie Cliniques, Groupe Hospitalier Jacques Monod - BP 24 - 76083 LE HAVRE Tél : 0232733223 - Fax : 0232733238

(Reçu le 4 octobre 2000 ; accepté le 25 octobre 2000)

\section{RÉSUMÉ}

Après un rappel concernant les aspects cliniques et thérapeutiques des syndromes cholinergiques et anticholinergiques, les molécules responsables de ces deux grands syndromes sont passées en revue. Organophosphorés et carbamates anticholinestérasiques, substances cholinomimétiques et molécules cholinergiques d'origine végétale pour le premier, antidépresseurs imipraminiques, certains antihistaminiques, des antiparkinsoniens, des antalgiques antispasmodiques, des préparations ophtalmiques, des plantes, ainsi que des champignons à alcaloïdes anticholinergiques pour le second. En urgence, l'analyse toxicologique du syndrome cholinergique consiste à mesurer les cholinestérases ; la recherche et le dosage du principe actif, permettant de confirmer la nature de l'intoxication, sont la chromatographie en phase liquide et la chromatographie en phase gazeuse couplées ou non à la spectrométrie de masse. En ce qui concerne le syndrome anticholinergique, seuls les antidépresseurs imipraminiques ont fait l'objet d'une approche analytique en urgence, en raison de la fréquence et de la gravité potentielle de ces intoxications. La réponse biologique pour valider ce syndrome utilise les mêmes techniques que celles de la confirmation du syndrome cholinergique.

\section{MOTS-CLÉS}

Cholinergique, anticholinergique, toxicité, dosage.

\section{SUMMARY}

After a reminder of the clinical and therapeutic aspects of the cholinergic and anticholinergic syndromes, the molecules responsible for these two major syndromes are reviewed. These include: organophosphorus and anticholinesterase carbamates, cholinomimetic substances and cholinergic drugs from plants and, imipraminic antidepressants, some antihistaminics, antiparkinson's antispasmodic, analgesics, ophthalmic preparations, plants including mushrooms with anticholinergic alkaloids for the first and the second syndrome, respectively. In emergency, toxicological analysis of the cholinergic syndrome consists in measuring the cholinesterases. Screening and quantitation methods to confirm the nature of the intoxication are liquid chromatography and gas chromatography coupled or not with mass spectrometry. With respect to the anticholinergic syndrome, only imipraminic antidepressants are subject of an analytical approach within the context of emergency, because of the high frequency and the potential gravity of these intoxications. The biological answer to validate this syndrome uses the same techniques as those for confirming cholinergic syndrome.

\section{KEY-WORDS}

Cholinergic, anticholinergic, toxicity, analysis. 


\section{Introduction}

Parmi les 150000 intoxications signalées chaque année aux Centres Anti Poisons Français, les médicaments sont en cause dans $50 \%$ des cas, les produits ménagers environ $15 \%$, les pesticides $4 \%$ et les végétaux moins de $3 \%$. Les symptômes cliniques sont souvent discrets voire inexistants.

Lors de certaines intoxications, on voit d'authentiques syndromes cholinergiques ou anticholinergiques. Bien que rares, ils peuvent être cependant mortels. Les syndromes cholinergiques résultent d'une importante accumulation d'acétylcholine dans les synapses du système nerveux central et périphérique alors que les syndromes anticholinergiques, ou atropiniques, résultent d'un blocage de la production d'acétylcholine dans ces mêmes synapses.

\section{Le Syndrome cholinergique Aspects cliniques et thérapeutiques}

Le syndrome cholinergique s'observe essentiellement lors d'une exposition aux insecticides organophosphorés et carbamates et après l'ingestion de certains champignons du genre clitocybe ou inocybe contenant de la muscarine. La fixation du toxique sur les cholinestérases, réversible pour les carbamates, souvent irréversible pour les organophosphorés, entraîne une accumulation d'acétylcholine responsable de manifestations cliniques : syndrome muscarinique, nicotinique et central. Quant à la muscarine contenue dans les champignons précités, elle a des propriétés parasympathicomimétiques puissantes.

Cliniquement, le syndrome cholinergique est donc constitué de l'intrication à des degrés divers des trois syndromes suivants :

- muscarinique ou sudorien, résultant de la stimulation du parasympathique post ganglionnaire : hypersécrétion salivaire, sudorale, digestive et bronchique ; action sur la musculature lisse avec myosis, bronchoconstriction, nausées, vomissements, augmentation du péristaltisme, miction et défécation involontaires ; bradycardie, hypotension artérielle, troubles de conduction et du rythme cardiaque pouvant entraîner un risque vital ;

- nicotinique, par dépolarisation persistante des muscles striés avec asthénie, paresthésies, fasciculations, crampes, parésies puis paralysies musculaires ; stimulation sympathique avec tachycardie, hypertension, hyperglycémie, hypokaliémie, hyperleucocytose ; - central avec euphorie, anxiété, agitation, céphalées, tremblements, confusion, ataxie, troubles de la conscience, parfois convulsions et coma convulsif.
Ces trois syndromes, selon leur intensité peuvent entraîner à tout moment une détresse respiratoire aiguë ou une atteinte cardiaque d'évolution mortelle ; mais le tableau clinique et sa gravité sont très variables suivant l'intensité de l'exposition d'où son grand polymorphisme.

Le diagnostic de ce syndrome cholinergique repose sur la notion d'inhalation, de contact cutané ou d'ingestion d'un insecticide organophosphoré ou carbamate, plus rarement de l'ingestion d'un champignon suspect, la survenue rapide, quelques minutes à quelques heures, de symptômes évocateurs associés entre eux et appartenant aux trois syndromes muscarinique, nicotinique et central et la mesure en urgence des cholinestérases plasmatiques. Mais lorsque l'anamnèse fait défaut, lorsque la symptomatologie est dissociée et qu'un ensemble de signes neurologiques, respiratoires ou digestifs dominent trop le tableau, ce diagnostic peut être difficile, retardé ou erroné.

Le traitement est décontaminateur ou évacuateur suivant la nature du toxique et sa voie d'entrée. Les mesures symptomatiques sont essentielles et visent à assurer le maintien des grandes fonctions vitales : neurologiques, cardiovasculaires, respiratoires, rénales et métaboliques. Il faut donc, suivant les cas, recourir à des méthodes plus ou moins invasives en milieu de réanimation pendant au minimum $24 \mathrm{~h}$ à $48 \mathrm{~h}$, délai au delà duquel il n'y a en général plus de risque vital.

Le traitement spécifique du syndrome cholinergique repose essentiellement sur l'utilisation de l'atropine qui antagonise les effets muscariniques mais n'a pas d'effet sur l'atteinte neuromusculaire et ne modifie pas les cholinestérases. Elle est administrée par voie intraveineuse de façon répétée (de 1 à $4 \mathrm{mg}$ toutes les 10 minutes chez l'adulte) et de fortes doses sont parfois nécessaires. L'atropinisation doit être poursuivie au moins $24 \mathrm{~h}$ et une surveillance très étroite est nécessaire.

Quant au pralidoxime, antidote permettant de régénérer les cholinestérases, il a un effet synergique avec l'atropine en cas d'intoxication par les organophosphorés, mais son action centrale est limitée. Son efficacité n'est pas établie dans les intoxications par insecticides carbamates.

\section{Les molécules du syndrome choliner- gique}

Il s'agit de substances très variées que l'on trouve dans des insecticides (organophosphorés ou carbamates), des médicaments alcaloïdes cholinergiques de synthèse et analogues de structure, des végétaux (champignọns à muscarine et alcaloïdes de diverses plantes), des armes chimiques (gaz de combat organophosphorés). 


\section{Organophosphorés anticholinestérasiques}

Excepté l'échothiopate, médicament du glaucome, ce sont pour l'essentiel des insecticides et des gaz de combat. Nous n'évoquerons que les composés présentant un intérêt pharmacologique ou toxicologique. La formule générale de ces produits a été définie par Schrader en 1952 (1).

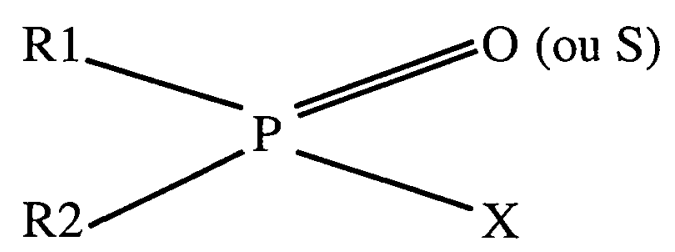

On distingue 4 groupes de composés :

- groupe $\mathrm{A}$ où $\mathrm{X}$ est un groupement halogène, cyanure ou thiocyanate ;

- groupe B où X est un groupement alkylthio, alkoxy ou arloxy ;

- groupe C dans lequel un soufre est substitué à l'oxygène dans la formule générale et $X$ est un groupement aryloxy, arylthio ;

- groupe D où X est un autre groupement phosphate ;

- groupe $\mathrm{E}$ où $\mathrm{X}$ est un ammonium quaternaire.

Les organophosphorés anticholinestérasiques présentent la propriété de se fixer et donc d'inhiber l'action des cholinestérases -ou estérases du groupe B- de nombreux organismes vivants (mammifères, insectes, nématodes, acariens). Si cette action est recherchée par éliminer de nombreux parasites, chez l'homme, elle peut être à l'origine d'intoxications graves. Selon la nature du produit et le degré d'exposition, les effets sont plus ou moins durables, de quelques minutes pour les moins toxiques, à plusieurs semaines pour les gaz de combat. Les organophosphorés anticholinestérasiques peuvent se fixer aux cholinestérases, mais également à d'autres estérases, cette fixation bloque la dégradation de l'acétylcholine et entraîne son accumulation au niveau de la plaque motrice et des synapses du système sympathique et du système nerveux central et explique leur toxicité. Certains composés, outre leur action inhibitrice de l'acétylcholinestérase ont également une action cholinergique directe. D'autres n'exercent leur action anticholinestérasique qu'après métabolisation ; ainsi le parathion contenant $\mathrm{P}=\mathrm{S}$ dans sa formule n'est pas toxique contrairement à son métabolite, le paraoxon possédant une fonction $\mathrm{P}=\mathrm{O}$. Pour les organophosphorés qui n'exercent leur action qu'après métabolisation, le tableau clinique de l'intoxication est donc retardé.

En ce qui concerne les organophosphorés gaz de combat, leur neurotoxicité est redoutable, les sujets pouvant être exposés à une dose létale en quelques minutes. La mort par hypoxie aiguë est la résultante de plusieurs mécanismes : obstruction des voies aériennes par le bronchospasme et la bronchorrhée (syndrome muscarinique), paralysie des muscles respiratoires (syndrome nicotinique), atteinte du système nerveux central avec crises convulsives, coma et dépression respiratoire (syndrome central). Les principaux gaz utilisés sont des agents du groupe $\mathrm{G}$ : le tabun ou GA (éthyldiméthylphosphoramidocyanidate), le sarin ou GB (isopropylméthyl-phosphonofluoridate) et le soman ou GD (pinacolylméthylphosphono-fluoridate) et le cyclohexylméthylphosphonofluoridate ou GF.

L'échothiopate (Phospholine Iodide ${ }^{\circledast}$ ), médicament du glaucome présente également une structure de type organophosphoré et exerce une action anticholinestérasique.

\section{Carbamates anticholinestérasiques}

Les carbamates anticholinestérasiques sont des esters de l'acide carbamique ou acide aminoformique $\mathrm{NH}_{2} \mathrm{COOH}$ et plus précisément de l'acide monométhyl ou diméthylcarbamique ; et répondent à la formule générale :

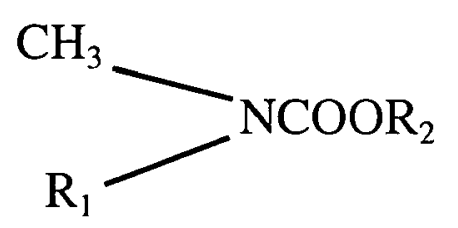

dans laquelle $\mathrm{R} 1$ est un hydrogène ou un méthyle et R2 un alcool, un phénol, une oxime. Ils présentent la caractéristique de réagir avec les cholinestérases par lesquelles il sont plus ou moins rapidement hydrolysés selon le même mécanisme que pour l'acétylcholine. Il s'agit d'un mode d'action comparable à celui observé avec les organophosphorés anticholinestérasiques mais la plus grande rapidité d'hydrolyse de la majorité des carbamates explique leur moindre toxicité. Ils sont utilisés comme insecticides. Les carbamates herbicides ou fongicides sont dénués d'activité anticholinestérasique. Quant aux carbamates anxiolytiques, ceux-ci n'exercent aucune action cholinergique. Les plus toxiques sont les dérivés de l'acide monométhylcarbamate, en particulier l'aldicarb et le carbofuran pour lesquels la dose létale $50\left(\mathrm{DL}_{50}\right)$ chez le rat est respectivement de 0,8 et $2,0 \mathrm{mg} / \mathrm{kg}$. D'autres moins toxiques comme le propoxur ou le carbaryl ont une $\mathrm{DL}_{50}$ respective chez le rat de 85 et $250 \mathrm{mg} / \mathrm{kg}$.

\section{Médicaments cholinergiques (anticholinestéra- siques ou non anticholinestérasiques)}

Il s'agit d'une molécule présentant une action parasympathomimétique cholinergique directe : la pilocarpine. Cet alcaloïde a été initialement extrait d'un petit arbuste d'Amérique du sud de la famille des rutacées : 
pilocarpus jaborandi. Aujourd'hui d'origine synthétique, c'est un médicament du glaucome, de la crise aiguë du glaucome et utilisé dans le diagnostic des causes de mydriase (11 collyres contiennent de la pilocarpine seule : Chibro-pilocarpine ${ }^{\circledast}$, Isoptopilocarpine $^{\circledast}$, Pilo-collyre ${ }^{\circledR}$, Pilocarpine Faure ${ }^{\circledast}$ ).

On trouve aussi des substances ayant une activité parasympathomimétique anticholinestérasique : les antimyasthéniques et un médicament du traitement symptomatique des troubles dyspeptiques chez l'adulte et chez l'enfant également parasympathomimétique inhibiteur de la cholinestérase : l'éséridine ou salicylate d'ésérine aminoxyde ou salicylate de physostigmine aminoxyde (Génésérine ${ }^{\circledR}$ ). Quatre antimyasthéniques anticholinestérasiques sont commercialisés en France : pyridostigmine bromure (Mestinon ${ }^{\circledR}$ ), ambénomium chlorure (Mytelase ${ }^{\circledR}$ ), néostigmine bromure (Néostigmine laphal ${ }^{\circledR}$ ), néostigmine méthylsulfate (Prostigmine $^{\circledast}$ ).

\section{Les autres molécules cholinergiques d'origine végétale :}

\section{- La muscarine}

C'est un alcaloïde que l'on rencontre dans divers champignons de la famille des Inocybes (I.) dont l'I. de patouillard, champignon que l'on rencontre couramment sous nos latitudes; ainsi que des clitocybes. Ces champignons sont dans $90 \%$ des cas à l'origine de syndromes sudoriens ou muscariniques mineurs (2), même si des observations mortelles ont été rapportées. Ces syndromes concernent environ $20 \%$ des intoxications par les champignons, en raison d'une confusion des espèces responsables avec des espèces comestibles comme les tricholomes (2). La muscarine se fixe sur les récepteurs de l'acétylcholine où elle exerce son action pharmacologique cholinergique. Il existe 8 muscarines; quatre isomères avec des formes lévogyres et dextrogyres (muscarine, épimuscarine, allomuscarine, épiallomuscarine) (2). Très souvent les champignons muscariniens contiennent également d'autres principes actifs, acétylcholine, choline, histamine en particulier, substances à l'origine de la variabilité de la symptomatologie clinique.

\section{- La pilocarpine}

Un certain nombre de plantes de la famille des rutacées, du genre pilocarpus, contiennent des alcaloïdes toxiques : pilocarpine, isopilocarpine, pilocarpidine, jaborine et jaboridine ; molécules qui possèdent toutes un noyau imidazole. Il s'agit principalement de pilocarpus microphyllus, et d'autres représentants mineurs : p. jaborandi, p. pennatifolius, p. trachylophus, p. racemosus.

\section{- L'arécoline}

L'arécoline est un alcaloïde cholinergique contenu dans les graines (noix d'Arec ou de Betel) d'un palmier areca catechu. Cette substance a été évaluée dans le traitement de la maladie d'Alzheimer. Elle est aujourd'hui réservée à l'usage vétérinaire comme anthelmintique cestodique.

\section{- L'ésérine ou physostigmine}

C'est le principal alcaloïde de la fève de Calabar provenant d'une plante de la famille des légumineuses : physostigma venenosum.

\section{Réponse analytique en urgence : les cho- linestérases}

Parmi les substances du syndrome cholinergique, un certain nombre de produits sont de puissants inhibiteurs des cholinestérases conduisant à une accumulation synaptique d'acétylcholine. Ce sont en particulier les insecticides organophosphorés et les gaz de combat organophosphorés et à un moindre degré les insecticides carbamates. Chez l'homme, il existe deux formes principales de cholinestérases, celles-ci diffèrent par leur localisation tissulaire, leur affinité de substrat, leur fonction physiologique : l'acétylcholinestérase (ACHE) et la butyrylcholinestérase, ou pseudocholinestérase (PCHE). Les principales caractéristiques et sources de variation de ces deux enzymes sont reportées tableau I. La diminution de l'activité cholinestérasique (ACHE ou PCHE) est en général bien corrélée avec l'intensité et la durée de l'exposition aux organophosphorés. Avec les carbamates insecticides, le dosage des cholinestérases n'a d'intérêt qu'à la phase aiguë de l'intoxication, en raison de la rapide réversibilité de l'action due à la destruction du principe actif. L'ACHE globulaire qui est la même molécule cible pour les substances anticholinestérasiques que celle trouvée dans les synapses constitue de ce fait un indicateur plus spécifique que la PCHE. Dans le cadre de la surveillance aux pesticides pour l'ACHE, la valeur limite recommandée est $70 \%$ de la valeur basale individuelle (American Conference of Govermental Industrial Hygienists - 1998, German Commission for the investigation of health hazards of chemical compounds in the work area - 1998, groupe de l'organisation mondiale de la santé - 1982). En cas d'intoxication grave, le retour à la normale de l'ACHE globulaire est beaucoup plus long que celui de la PHCE plasmatique. Dans les intoxications aiguës seulement, il existe une assez bonne corrélation entre le degré d'inhibition de l'ACHE et la symptomatologie clinique (tableau II).

Une diminution, voire un effondrement des cholinestérases peut survenir à la suite d'une anesthésie aux 
Tableau I : Cholinestérases chez l'homme : principales caractéristiques selon Maroni (3).

\begin{tabular}{|c|c|c|c|}
\hline \multicolumn{2}{|c|}{ E } & ACHE & PCHE \\
\hline \multicolumn{2}{|l|}{ Localisation } & $\begin{array}{l}\text { Globules rouges } \\
\text { Tissu nerveux }\end{array}$ & $\begin{array}{l}\text { Plasma } \\
\text { Foie } \\
\text { Cellules gliales }\end{array}$ \\
\hline \multicolumn{2}{|l|}{ Substrat } & Acétylcholine & $\begin{array}{l}\text { Butyrylthiocholine } \\
\text { Succinylcholine } \\
\text { Acétylcholine }\end{array}$ \\
\hline \multicolumn{2}{|c|}{ Variations intra et interindividuelles } & $3-7 \% ; 10-18 \%$ & $6 \% ; 15-25 \%$ \\
\hline \multicolumn{2}{|l|}{ Variations selon le sexe } & NON & $\begin{array}{l}\text { Valeurs de l'adules à } 6 \text { mois : } \\
\text { > } 15 \text { à } 20 \% \text { femmes < } 39 \text { ans } \\
\text { enceintes sans contraception } \\
\text { \ } 30 \% \text { femmes enceintes < } 41 \text { ans ou } \\
\text { sous contraception orale }\end{array}$ \\
\hline \multicolumn{2}{|c|}{$\begin{array}{l}\text { Variations selon la masse corporelle et } \\
\text { la cholestérolémie }\end{array}$} & NON & OUI \\
\hline \multicolumn{2}{|l|}{ Variations raciales } & NON & 4 chez le noir \\
\hline \multirow[t]{2}{*}{ Variations en pathologie } & $y$ & $\begin{array}{l}\text { Leucémies } \\
\text { Cancers }\end{array}$ & $\begin{array}{l}\text { Atteintes hépatiques } \\
\text { Insuffisance rénale } \\
\text { Insuffisance cardiaque } \\
\text { Cancers } \\
\text { Réactions allergiques } \\
\text { Infections aiguës } \\
\text { Anémies }\end{array}$ \\
\hline & $\pi$ & $\begin{array}{l}\text { Polycythémie } \\
\text { Thalassémie } \\
\text { Autres dyscrasies sanguines congénitales }\end{array}$ & $\begin{array}{l}\text { Hyperthyroïdie } \\
\lambda \text { métabolisme basal }\end{array}$ \\
\hline
\end{tabular}

Tableau II : Sévérité et pronostic de l'intoxication aiguë par organophosphoré en fonction du degré d'inhibition de l'Acétylcholinestérase (ACHE) [selon Maroni (3)].

\begin{tabular}{|c|c|c|c|}
\hline $\begin{array}{l}\text { \% d'inhibition } \\
\text { de l'ACHE }\end{array}$ & $\begin{array}{c}\text { Degré } \\
\text { d'intoxication }\end{array}$ & Signes cliniques & Pronostic \\
\hline $50-60$ & Faible & $\begin{array}{l}\text { Fatigue, céphalées, vertiges, nausées salivation, } \\
\text { larmoiement, myosis, spasme bronchique modéré }\end{array}$ & Récupération 1 à 3 jours \\
\hline $60-90$ & Modéré & $\begin{array}{l}\text { Fatigue brutale, troubles visuels, sialorrhée, } \\
\text { sudation, vomissement, diarrhée, bradycardie, } \\
\text { hypertonie, tremblements, troubles de la marche, } \\
\text { myosis }\end{array}$ & Récupération 1 à 2 semaines \\
\hline $90-100$ & Sévère & $\begin{array}{l}\text { Tremblements soudains, convulsions généralisées, } \\
\text { troubles psychiques, cyanose intense, } \\
\text { œdème pulmonaire, coma }\end{array}$ & $\begin{array}{l}\text { Risque de décès consécutif à } \\
\text { une insuffisance cardiaque } \\
\text { ou respiratoire }\end{array}$ \\
\hline
\end{tabular}


curarisants soit par surcharge en curares soit en raison d'un déficit congénital en cholinestérase. L'examen biochimique à l'aide d'inhibiteurs réversibles permet de déterminer le phénotype de la cholinestérase déficiente.

Pour les modalités pratiques des dosages de l'acétylcholinestérase et de la butyrylcholinestérase, se reporter à la fiche de toxicologie hospitalière "activité anticholinestérasique" du COFRAC (Comité Français d'Accréditation).

\section{L'analyse toxicologique dans les syn- dromes cholinergiques}

Elle permet de confirmer la nature des molécules à l'origine du syndrome cholinergique et de s'assurer qu'il n'y a pas d'autre substance associée.

\section{Organophosphorés pesticides et carbamates pes- ticides}

L'exploration analytique des intoxications par les pesticides faisant l'objet d'un autre article de ce numéro (5), cet aspect ne sera pas développé, cependant nous suggérons la lecture d'une très récente revue (3). En ce qui concerne la partie analytique, nous recommandons vivement la méthode de Lacassie et coll. (6). Elle permet la recherche simultanée et le dosage dans les milieux biologiques de 61 pesticides grâce à de nouveaux supports d'extraction en phase solide. Une première extraction sélective est réalisée en présence d'un étalon interne pour 47 pesticides volatiles (dont 29 organophosphorés), l'analyse est effectué par chromatographie en phase gazeuse couplée à la spectrométrie de masse $(\mathrm{CG} / \mathrm{SM})$. Les limites de détection $(2,5$ à $50 \mu \mathrm{g} / \mathrm{l})$ et de quantification $(5$ à $100 \mu \mathrm{g} / \mathrm{l})$ sont suffisantes dans le cadre de la toxicologie clinique. Une seconde extraction sélective, puis mesure par chromatographie liquide couplée à la spectrométrie de masse $\mathrm{CL} / \mathrm{SM}$, est appliquée aux composés polaires, au nombre de 14 dont 11 carbamates, avec des limites de détection et de quantification identiques à celles obtenues par CG/SM pour les organophosphorés.

\section{Organophosphorés gaz de combat}

Le dosage de ces molécules, tabun, sarin, soman et produits apparentés, fait le plus souvent appel à la CG/SM. $\mathrm{La}$ réalisation technique est toujours délicate pour trois raisons : il s'agit de mesurer des concentrations extrêmement faibles, la préparation de l'échantillon est susceptible d'induire une dégradation des molécules à doser, la conservation des principes actifs dans les milieux biologiques est mauvaise. On distingue deux groupes de méthodes : celles qui permettent de quantifier directement les produits parents et celles qui mesurent les principaux métabolites qui sont des acides alkyméthylphosphoniques. Les premières font appel à la CG/SM en mode sélection d'ions ou couplée à la haute résolution pour les dosages sanguins $(7,8)$. Les secondes consistent à doser les acides alkyméthylphosphoniques après dérivation puis CG/MS en mode courant ionique total ou en mode sélection d'ions et peuvent être appliquées au sang, aux urines, à la salive, voire à des organes $(9,10,11)$. Dans ce second groupe, l'utilisation de l'ionisation chimique négative couplée à la spectrométrie de masse $(\mathrm{CG} / \mathrm{SM} / \mathrm{ICN})$ permet d'abaisser considérablement la limite de détection : $60 \mathrm{pg} / \mathrm{ml}$ contre $5 \mathrm{ng} / \mathrm{ml}$ en mode sélection d'ions et $50 \mathrm{ng} / \mathrm{ml}$ en mode courant ionique total (10). Des techniques enzymatiques pour la détermination sanguine du soman ont été proposées $(12,13)$. En ce qui concerne la CL/SM, celle-ci n'est utilisable que pour des dosages dans l'eau et n'est pas applicable aux milieux biologiques (14).

\section{Médicaments cholinergiques anticholinestéra- siques}

$\rightarrow$ Médicaments antimyasthéniques ou ammonium quaternaires anticholinestérasiques

La néostigmine, la pyridostigmine et l'ambenomium (15) peuvent être quantifiés dans les milieux biologiques par CL/BD et par CL/SM. En ce qui concerne la néostigmine et la pyridostigmine, les demi-vies d'élimination sont respectivement comprises entre $0,4-1,3$ heures et entre $0,4-1,9$ heures ; les volumes de distribution s'établissent entre 0,1 et $1,1 \mathrm{l} / \mathrm{kg}$ et entre 0,5 et $1,8 \mathrm{l} / \mathrm{kg}$; et les valeurs thérapeutiques s'échelonnent entre 1 et $10 \mathrm{ng} / \mathrm{ml}$ ou $\mu \mathrm{g} / \mathrm{l}$; et entre 40 et $60 \mathrm{ng} / \mathrm{ml}$ (16).

$\rightarrow$ Autres médicaments cholinergiques anticholinestérasiques

- Eséridine (Generesine ${ }^{\circledR}$ )

Source rare d'intoxication, l'éséridine est classiquement identifiée et mesurée dans les milieux biologiques par CL/BD.

- Ecothiopate (Phospholine iodide ${ }^{\circledR}$ )

Il n'a pas fait l'objet d'études analytiques du fait de l'absence d'intoxications documentées. Il n'existe que sous forme de collyre et chaque flacon contient $0,9 \mathrm{mg}$ de principe actif.

\section{Médicaments cholinergiques non anticholinesté- rasiques}

$\rightarrow$ Pilocarpine et dérivés 
La pilocarpine est caractérisée par une demi-vie d'élimination de 1,7 heure et un volume de distribution de $2,71 / \mathrm{kg}$ (16). Les concentrations thérapeutiques sont comprises entre 1 et $42 \mathrm{ng} / \mathrm{ml}$ (17). Le dosage de la pilocarpine, dans le cadre thérapeutique, peut être réalisé par CL/BD. Pour une prise d'essai de $0,5 \mathrm{ml} \mathrm{la}$ limite de quantification est de $10 \mathrm{ng} / \mathrm{ml}$ (18). Une technique de détermination de la pilocarpine et de l'acide pilocarpique après dérivation et CG/SM a été proposée (19). La différenciation des isomères de la pilocarpine et de l'acide pilocarpique peut être réalisée par chromatographie liquide couplée à une double détection de masse (CL/SM/SM) avec une limite de détection voisine du $\mathrm{ng} / \mathrm{ml}(20)$.

\section{La muscarine}

Si la muscarine peut être mise en évidence dans les champignons qui en contiennent (21), sa recherche dans les milieux biologiques est extrêmement difficile. Pourtant celle-ci serait très utile en raison de la grande toxicité de cette molécule. $\mathrm{La} \mathrm{DL}_{50}$ par voie intraveineuse chez la souris est de $0,23 \mathrm{mg} / \mathrm{kg}$. Nous avons eu un cas d'intoxication mortelle par inocybe de patouillard, la recherche dans le sang par CL/SM n'a pas permis de détecter la muscarine.

\section{Analyse toxicologique des autres alcaloüdes végé- taux cholinergiques}

En raison des concentrations très faibles trouvées dans les milieux biologiques, elle fait appel à la CL/SM. A l'occasion d'une revue, Gaillard (22) propose une technique très originale d'identification et de dosage de 24 alcaloïdes végétaux et composés cyanogénétiques dans les intoxications par les plantes. Une revue bibliographique comportant les principes actifs, la toxicologie analytique et les cas d'intoxications chez l'homme pour 40 espèces végétales principales ainsi que de nombreuses espèces apparentées est également présentée. En ce qui concerne la technique proposée, elle permet à partir de $2 \mathrm{ml}$ d'échantillon (sang total, plasma) soumis à une triple extraction de quantifier 5 alcaloïdes végétaux par CL/SM parmi ceux qui nous intéressent : - 3 alcaloïdes cholinergiques : arécoline, ésérine, pilocarpine

- 2 alcaloïdes anticholinergiques : atropine, scopolamine.

Le tableau III montre pour les 5 molécules citées les excellentes performances de la méthode, en totale adéquation avec les exigences de la toxicologie clinique.

\section{Le Syndrome anticholiner- gique}

\section{Aspects cliniques et thérapeutiques}

Le syndrome anticholinergique ou atropinique est produit par des substances de type atropine qui inhibent les effets de la stimulation de la fibre post ganglionnaire du parasympathique et possèdent à doses élevées une action excitatrice centrale. Elles s'opposent donc, par un blocage compétitif et réversible des récepteurs périphériques et centraux, à l'action de l'acétylcholine. Elles inhibent l'effet bradycardisant de celle-ci, ont une action antispasmodique au niveau des fibres musculaires lisses du tube digestif, des voies biliaires et urinaires, ralentissant la vidange gastrique et diminuant les sécrétions digestives, lacrymales et sudorales.

Les signes et symptômes du syndrome anticholinergique qui sont liés à un blocage périphérique sont mydriase, sécheresse de la peau et des muqueuses, vasodilatation, hyperthermie, rétention urinaire, iléus et

Tableau III : Étude de la reproductibilité quotidienne, rendement et limite de détection $(L D)$ des alcaloïdes $(n=10)$ par CLSSM selon Gaillard (22).

\begin{tabular}{|c|c|c|c|c|c|c|}
\hline \multirow[t]{2}{*}{ Composé } & \multicolumn{4}{|c|}{ Coefficient de variation $(\%)$ à } & \multirow{2}{*}{$\begin{array}{c}\text { Rendement } \\
\text { d'extraction }(\%)\end{array}$} & \multirow{2}{*}{$\begin{array}{r}\text { LD } \\
\text { (ng/m }\end{array}$} \\
\hline & $0,1 \mathrm{ng} / \mathrm{ml}$ & $1 \mathrm{ng} / \mathrm{ml}$ & $10 \mathrm{ng} / \mathrm{ml}$ & $100 \mathrm{ng} / \mathrm{ml}$ & & \\
\hline Arécoline & - & 19,0 & 8,0 & 5,6 & 63,1 & 0,91 \\
\hline Atropine & 11,8 & 6,1 & 4,2 & 3,7 & 70,9 & 0,09 \\
\hline Esérine & - & 17,2 & 8,7 & 8,0 & 103,7 & 0,17 \\
\hline Pilocarpine & - & - & 7,6 & 6,6 & 68,1 & 1,07 \\
\hline Scopolamine & - & 12,4 & 9,1 & 6,9 & 64,4 & 0,31 \\
\hline
\end{tabular}


tachycardie. Les signes de blocage central sont confusion, agitation, hallucinations, myoclonies, tremblements, convulsions, coma et dépression respiratoire. La gravité du tableau clinique est très variable, dépendant de la nature du toxique en cause et de la quantité ingérée. Les formes bénignes sont les plus fréquentes, se limitant à une mydriase, une sécheresse buccale et une tachycardie sinusale, alors que les formes graves sont déterminées par la présence de signes neurologiques parfois dangereux sur le plan vital. Une confusion mentale, des hallucinations, suivie d'un coma convulsif associé à une mydriase doit faire évoquer un syndrome anticholinergique grave.

Les toxiques le plus souvent en cause sont les antidépresseurs tricycliques, certains antihistaminiques, antiparkinsoniens, antipsychotiques, antispasmodiques, myorelaxants, mais aussi préparations ophtalmiques. Les alcaloïdes de la belladone contenus dans des végétaux comme la belladone, les morelles, la douce-amère et des champignons appartenant au groupe des amanites peuvent provoquer des intoxications sévères avec signes cardiaques et neurologiques. Le diagnostic est dans l'ensemble facile à évoquer ; les signes ont parfois une bonne valeur d'orientation étiologique. Le traitement est symptomatique : hydratation, sédation, prévention ou traitement des convulsions, mesures de réanimation dans les cas les plus graves.

La physostigmine, inhibiteur réversible des cholinestérases, a un effet sur l'encéphalopathie et la tachycardie mais son action est fugace et son usage controversé en raison d'effets secondaires parfois dangereux. Elle peut être recommandée avec prudence dans certaines formes graves : agitation extrême, coma convulsif, tachycardie supra ventriculaire sévère, mais elle n'est pratiquement pas utilisée en France.

\section{Les molécules du syndrome anticholi- nergique}

Comme pour le syndrome cholinergique, les substances responsables du syndrome anticholinergique sont très variées. On trouve essentiellement des médicaments appartenant à des classes pharmacologiques diverses, ainsi que des végétaux.

\section{Antidépresseurs imipraminiques anticholiner- giques}

Ce groupe comprend 9 principes actifs : amitriptyline $\left(\right.$ Laroxy $^{\oplus}{ }^{\oplus}$, Elavil $\left.{ }^{\oplus}\right)$, amoxapine (Défanil $\left.{ }^{\circledR}\right)$, clomipramine Anafranil $^{\oplus}$, Clomipramine $^{\oplus}$ ), désipramine $\left(\right.$ Pertofran $^{\oplus}$ ), dosulépine (Prothiaden ${ }^{\oplus}$ ), doxépine (Quitaxon ${ }^{\oplus}$ ), imipramine (Tofranil $\left.{ }^{\oplus}\right)$, maprotiline $\left(\right.$ Ludiomil $\left.^{\circledR}\right)$, trimipramine (Surmontil $\left.{ }^{\oplus}\right)$; commerciali- sés sous 36 spécialités différentes au dictionnaire Vidal électronique (23). Les effets indésirables découlent pour la plupart des propriétés pharmacologiques des antidépresseurs imipraminiques. Liés aux effets périphériques de la molécule, on note en particulier, une action anticholinergique (par ordre de fréquence décroissante) : sécheresse de la bouche, constipation, troubles de l'accommodation, tachycardie, sueurs, troubles de la miction et éventuellement rétention urinaire (23).

\section{Antihistaminiques anticholinergiques}

Dans la catégorie des antihistaminiques $\mathrm{H}_{1}$ on distingue des antihistaminiques non anticholinergiques et des antihistaminiques anticholinergiques. Cette dernière classe comprend 10 substances actives : alimémazine (Théralène $\left.{ }^{\circledast}\right)$, bromphéniramine (Dimégan $\left.{ }^{\circledR}\right)$, buclizine $\left(\right.$ Aphilan $^{\oplus}$ ), carbinoxamine (Allergefon ${ }^{\oplus}$ ), cyproheptadine (Périactine ${ }^{\circledast}$ ), dexchlor-phéniramine (Polaramine ${ }^{\circledast}$ ), doxylamine (Mépéridine ${ }^{\circledast}$ ), hydroxyzine $\left(\right.$ Atarax $\left.^{\oplus}\right)$, méquitazine (Primalan ${ }^{\oplus}$, Quitadrill $\left.{ }^{\circledR}\right)$, prométhazine (Phénergan ${ }^{\circledast}$ ) ; on compte 28 spécialités inscrites au Vidal (23). Ces molécules exercent toutes un effet anticholinergique à l'origine d'effets indésirables périphériques (23).

\section{Antiparkinsoniens anticholinergiques}

Cette classe de médicaments comporte 3 principes actifs : bipéridène (Akinéton ${ }^{\circledast}$ ), trihexyphénidyle (Artane $^{\oplus}$, Parkinane ${ }^{\circledR}$ ), tropatépine $\left(\right.$ Lepticur ${ }^{\oplus}$ ) ; on trouve 12 spécialités dans le dictionnaire Vidal électronique (23). Les antiparkinsoniens anticholinergiques montrent à la fois une action anticholinergique centrale et périphérique donnant en cas de surdosage les symptômes de l'intoxication aiguë par les anticholinergiques : mydriase, agitation, confusion mentale, hallucinations, convulsions, hyperventilation, tachycardie et hyperthermie (23).

\section{Antipsychotiques neuroleptiques phénothiazi- niques anticholinergiques}

Ce groupe comprend 10 substances actives : chlorpromazine (Largactil $\left.{ }^{\circledR}\right)$, cyamémaine $\left(\right.$ Tercian $\left.^{\circledR}\right)$, fluphénazine (Modécate ${ }^{\oplus}$, Moditem $^{\oplus}$ ), lévomépromazine $\left(\right.$ Nozinan $\left.^{\oplus}\right)$, perphénazine énantate (Trilifan retard $\left.^{\oplus}\right)$, pipotiazine $\left(\right.$ Piportil $\left.^{\oplus}\right)$, propériciazine $\left(\right.$ Neuleptil $\left.^{\oplus}\right)$, thiopropérazine (Majeptil ${ }^{\oplus}$ ), thioridazine (Melleril ${ }^{\oplus}$ ), trifluopérazine (Terfluzine ${ }^{\circledast}$ ) ; disponibles sous 46 spécialités au Vidal électronique (23). Outre les propriétés antidopaminergiques de ces molécules, elles présentent également des effets antihistaminiques, adrénolytiques et anticholinergiques marquées. 


\section{Antalgiques-antispasmodiques anticholinergiques}

Cette catégorie est essentiellement représentée par les alcaloïdes de la belladone produits par synthèse ainsi que des dérivés. Ce sont des antispasmodiques/antalgiques anticholinergiques à visée digestive et génitourinaire pour certains : l'atropine sulfate qui regroupe 29 spécialités et qui est également un médicament des urgences cardiovasculaires, la scopolamine (2 spécialités), l'atropine $\mathrm{N}$-oxyde (Génatropine ${ }^{\circledR}$ ), le prifinium $\left(\right.$ Riabal $\left.^{\circledR}\right)$, la dihexyvérine (Spasmodex $\left.{ }^{\oplus}\right)$. Le tiémonium (Viscéralgine ${ }^{\circledast}$ ) antispasmodique mixte musculotrope et anticholinergique n'exerce qu'un cinquantième de l'effet de l'atropine à concentration moléculaire identique (23).

\section{Préparations ophtalmiques anticholinergiques}

Ce sont des collyres mydriatiques atropiniques représentés par 10 spécialités à base de sulfate d'atropine, 2 collyres contenant du cyclopentolate $\left(\right.$ Skiacol $\left.^{\oplus}\right), 2$ autres de 1'homatropine (Homatropine ${ }^{\circledR}$, Isopto-homatropine ${ }^{\oplus}$ ), une de l'hyoscyamine (Duboisine $^{\circledR}$ ) et 3 du tropicamide (23).

\section{Plantes à alcaloüdes anticholinergiques}

Il s'agit d'alcaloïdes contenus dans des plantes de la famille des solanacées (22). La belladone (atropa belladona) est la plus fréquente sous nos latitudes, la teneur en alcaloïdes est comprise entre $0,3 \%$ et $1 \%$, la Lhyoscyamine et l'atropine ou DL-hyoscyamine représentant 90 à $95 \%$ des alcaloïdes totaux et la scopolamine 5 à $10 \%$. La jusquiame noire (hyoscyamus niger), un peu moins toxique que la belladone, contient de 0,2 à $0,5 \%$ d'alcaloïdes, surtout sous forme d'hyoscyamine, mais également sous forme d'atropine et de scopolamine. Dans le datura (datura stramonium) et des espèces voisines, la concentration en alcaloïdes oscille entre 0,2 et $0,6 \%$, la proportion d'LHyoscyamine et d'atropine est voisine de $67 \%$ des alcaloïdes et celle de scopolamine proche de $33 \%$. Les fleurs séchées de datura metel appelées yangjinhua (85\% de scopolamine et $15 \%$ d'hyoscyamine et d'atropine) appartiennent aux remèdes végétaux chinois, utilisées dans le traitement de l'asthme, de la bronchite chronique, de douleurs et symptômes divers, sont à l'origine d'intoxications (24). Autre solanacée, la mandragore (mandragora officinarum) contient essentiellement de la scopolamine. Toujours dans les solanacées, des plantes appartenant à la famille des morelles peuvent être à l'origine d'un syndrome anticholinergique atypique : la douce amère (solanum dulcamara), la morelle noire (solanum nigrum). Elles contiennent deux classes de glycoalcaloïdes toxiques : les solanines et les chaconines présentant un noyau stéroïdique commun, la solanidine (22).

\section{Champignons à principes actifs anticholinergiques}

Des champignons appartenant à l'espèce des amanites peuvent être à l'origine d'un syndrome mycoatropinique ou panthérinien. Parmi ceux-ci, l'amanite muscarine (Amanita muscaria) contient les acides distizolopique et stizolopilique à des concentrations qui pourraient être responsables d'effets cliniques de type anticholinergique (mydriase, tachycardie), ces composés ayant des analogies avec les produits d'oxydation de la L-Dopa (2).

\section{Réponse analytique en urgence}

Parmi les molécules anticholinergiques, seuls les antidépresseurs ont fait l'objet d'études concernant la réponse analytique en urgence.

\section{Antidépresseurs imipraminiques anticholiner- giques}

Les intoxications par ces molécules constituent une préoccupation majeure pour les cliniciens. En effet, il est admis qu'une ingestion de $500 \mathrm{mg}$ de principe actif est potentiellement toxique et $1 \mathrm{~g}$ peut avoir une conséquence mortelle. La relative fréquence des intoxications médicamenteuses par les antidépresseurs imipraminiques, en seconde position après celles par les benzodiazépines (25), majore les problèmes de prise en charge de ces malades (26). Dans le cadre de l'urgence, trois stratégies sont proposées :

- Dépistage immunologique semi-quantitatif et confirmation par chromatographie liquide

Partant du principe que parmi les antidépresseurs, les dérivés imipraminiques, les plus toxiques, sont très bien détectés par l'analyse immunologique, dès 1993 nous avons proposé avec nos collègues réanimateurs $(25,27,28)$ une stratégie qui a été validée. Les malades sont surveillés en milieu spécialisé si l'un des trois critères suivants est positif : défaillance vitale, troubles électriques à l'ECG, concentration plasmatique supérieure à $500 \mu \mathrm{g} / \mathrm{l}$ exprimée en imipramine. Secondairement, chaque dépistage fait l'objet d'un contrôle systématique par CL/BD, mais ce dernier n'est réalisé que les jours et heures ouvrables, la quantification des principes actifs et métabolites n'étant effectuée qu'après entente avec l'équipe clinique (28). Les principales données pharmacocinétiques sont reportées tableau IV.

- Recherche et dosage par chromatographie en phase gazeuse couplée à la spectrométrie de masse

Il s'agit d'une méthode de recherche et de dosage spécifique et sensible des antidépresseurs imipraminiques et de certains de leurs métabolites. Plusieurs techniques ont été proposées $(29,30,31)$. Certains laboratoires réalisent 
Annales de Toxicologie Analytique, vol. XII, n4, 2000

Tableau IV : Principales caractéristiques pharmacocinétiques des antidépresseurs imipraminiques selon Wennig (16), Uges (36), Repetto (37) et Meyer (38).

\begin{tabular}{|c|c|c|c|c|c|}
\hline \multirow[t]{2}{*}{ Principe actif } & \multirow[t]{2}{*}{ Métabolite } & \multirow{2}{*}{$\begin{array}{c}\text { Demi-vie } \\
\text { d'élimination (h) }\end{array}$} & \multirow{2}{*}{$\begin{array}{c}\text { Vd } \\
(1 / \mathrm{kg})\end{array}$} & \multicolumn{2}{|c|}{$\begin{array}{l}\text { Concentrations plasmatiques } \\
\text { (ng/ml ou } \mu \mathrm{g} / \mathbf{l})\end{array}$} \\
\hline & & & & Thérapeutiques & Toxiques \\
\hline Amitriptyline & Nortriptyline & $\begin{array}{l}10-30 \\
22-68\end{array}$ & $\begin{array}{l}12-16 \\
17-31\end{array}$ & $\begin{array}{l}50-200 \\
50-250\end{array}$ & $\begin{array}{l}>500 \\
>500\end{array}$ \\
\hline Amoxapine & & $8-30$ & $11-48$ & $200-400$ & \\
\hline Clomipramine & Desméthylclomipramine & $20-40$ & $17-25$ & $\begin{array}{c}20-150 \\
100-200\end{array}$ & $\begin{array}{l}500-600 \\
300-400\end{array}$ \\
\hline Désipramine & & $10-30$ & $18-42$ & $30-300$ & $>500$ \\
\hline Dosulépine & Nordosulépine & $\begin{array}{l}11-40 \\
22-60\end{array}$ & $20-92$ & $\begin{array}{c}50-200 \\
100-200\end{array}$ & $\begin{array}{c}>800 \\
500-750\end{array}$ \\
\hline Doxépine & Nordoxépine & $\begin{array}{c}8-25 \\
33-80\end{array}$ & $9-33$ & $\begin{array}{c}50-250 \\
100-250\end{array}$ & $\begin{array}{l}>500 \\
>500\end{array}$ \\
\hline Imipramine & Désipramine & $\begin{array}{c}6-26 \\
10-30\end{array}$ & $\begin{array}{l}28-61 \\
18-42\end{array}$ & $\begin{array}{l}50-300 \\
30-300\end{array}$ & $\begin{array}{l}>500 \\
>500\end{array}$ \\
\hline Maprotiline & Desméthylmaprotiline & $20-60$ & $23-70$ & $\begin{array}{c}50-250 \\
100-400\end{array}$ & $\begin{aligned}> & 500 \\
750 & -1000\end{aligned}$ \\
\hline Trimipramine & & $10-40$ & 31 & $20-200$ & $>500$ \\
\hline
\end{tabular}

cette surveillance y compris en garde (29). Cette démarche présente outre la spécificité, l'avantage de permettre le dosage d'antidépresseurs non imipraminiques. En ce qui concerne les performances analytiques, la limite de détection est comprise entre 10 et $50 \mathrm{ng} / \mathrm{ml}$ et la limite de quantification entre 20 et $100 \mathrm{ng} / \mathrm{ml}$.

- Recherche par chromatographie liquide haute performance couplée à une barrette de diodes

Il existe un ensemble commercialisé par la société Biorad (Vitry-sur-Seine, France) depuis plusieurs années : le système Remedi ${ }^{\oplus}$. C'est un chromatographe liquide haute pression entièrement automatisé utilisant quatre colonnes en série permettant la purification de l'échantillon et la séparation des composés. La détection est réalisée par l'acquisition continue des spectres des substances éluées entre $193 \mathrm{~nm}$ et $305 \mathrm{~nm}$. L'identification des substances se fait par la comparaison des spectres obtenus avec ceux d'une bibliothèque comportant plus de 500 molécules neutres ou basiques. L'intérêt de ce système repose sur sa rapidité - la durée d'un examen est de 20 minutes comprenant la préparation automatique de l'échantillon - mais également sur sa praticabilité et sur le nombre de molécules et métabolites identifiables. Il a été l'objet de plusieurs évaluations favorables $(32,33,34)$. L'appareil est bien adapté au dépistage en urgence des intoxications par les antidépresseurs imipraminiques anticholinergiques, mais pas à leur quantification. Un autre système, Alliance ${ }^{\circledast}$ commercialisé par la société Waters (Saint Quentin en Yvelines, France) présente des caractéristiques similaires mais la préparation de l'échantillon est manuelle, ce qui augmente considérablement le temps d'analyse (35). La bibliothèque contient 970 molécules et métabolites. Il est bien adapté aux dosages de ces molécules.

\section{L'analyse toxicologique dans les syn- dromes anticholinergiques}

\section{Antidépresseurs imipraminiques anticholiner- giques}

Les techniques spécifiques décrites dans le précédent chapitre et traitant de la réponse analytique en urgence peuvent être a fortiori utilisées en routine, CG/SM $(29,30)$ et CL/BD $(28)$. Le Remedi ${ }^{\oplus}$ n'est pas adapté à la quantification de ces molécules dans ce cadre.

\section{Antihistaminiques anticholinergiques}

La plupart de ces molécules, dont les caractéristiques pharmacocinétiques sont indiquées tableau V, sont faci- 
Tableau V : Principales caractéristiques pharmacocinétiques des antihistaminiques anticholinergiques selon Wennig (16).

\begin{tabular}{|c|c|c|c|c|}
\hline \multirow[t]{2}{*}{ Principe actif } & \multirow{2}{*}{$\begin{array}{c}\text { Demi-vie } \\
\text { d'élimination (h) }\end{array}$} & \multirow{2}{*}{$\begin{array}{c}\text { Vd } \\
(1 / k g)\end{array}$} & \multicolumn{2}{|c|}{$\begin{array}{c}\text { Concentrations plasmatiques } \\
\qquad(\mathrm{ng} / \mathrm{ml} \text { ou } \mu \mathrm{g} / \mathrm{l})\end{array}$} \\
\hline & & & Thérapeutiques & Toxiques \\
\hline Alimémazine & $3,6-7$ & - & $50-400$ & $>500$ \\
\hline Bromphéniramine & $15-22$ & $2,5-10$ & $\begin{array}{c}\text { Sérum : } 8-16 \\
\text { Sang total }: 8-150\end{array}$ & $>1000$ \\
\hline Chlorphéniramine & $15-30$ & $1-10$ & $8-16$ & $>1000$ \\
\hline Cyproheptadine & - & - & 30 & - \\
\hline Doxylamine & 10 & - & $70-150$ & $>700$ à > 1200 \\
\hline Hydroxyzine & $2,5-20$ & 22,5 & $\begin{array}{c}50-90 \\
\text { (pic) }\end{array}$ & $>100$ \\
\hline Prométhazine & $7-14$ & $9-18$ & $100-400$ & $>1000$ \\
\hline
\end{tabular}

lement quantifiées par $\mathrm{CL}$ ou par CG/SM $(39,40,41,42,43,44,45)$. Des techniques alternatives ont également été décrites par CL/SM/SM $(46,47)$.

\section{Antiparkinsoniens anticholinergiques}

Ces médicaments sont classiquement dosés par CL/BD même si des techniques de CG/SM et acquisition en mode sélection d'ions (48), d'électrophorèse capillaire (49), ou de CL/SM qui permet de quantifier les énantiomères $(47,50)$, ont été proposées. Pour le bipéridène, dont la demi-vie est de 18 à 24 heures, les concentrations thérapeutiques sont comprises entre 50 et 100 $\mathrm{ng} / \mathrm{ml}$ (38). En ce qui concerne le trihexyphénidyle, de demi-vie 3 à 7 heures, les valeurs usuelles et toxiques valent respectivement 50 à $200 \mathrm{ng} / \mathrm{ml}$ et supérieures à $500 \mathrm{ng} / \mathrm{ml}(16,36,38)$. Quant à la tropatépine, les études de pharmacocinétique chez le volontaire sain révèlent une demi-vie voisine de 40 heures et des concentrations thérapeutiques de 10 à $30 \mathrm{ng} / \mathrm{ml}$ (51). Dans un cas hospitalier d'intoxication mortelle par cette molécule, nous avons pu mettre en évidence outre une concentration élevée $(139 \mathrm{ng} / \mathrm{ml})$, la présence d'un métabolite par $\mathrm{CL} / \mathrm{BD}$ (observation non publiée).

\section{Antipsychotiques neuroleptiques phénothiazi- niques anticholinergiques}

Il s'agit de substances qui sont le plus souvent mesurées par CL/BD dont les performances sont suffisantes pour les besoins de la toxicologie hospitalière, même si la $\mathrm{CG} / \mathrm{SM}$ peut être mise en œuvre pour la plupart d'entre elles. Les principales données pharmacocinétiques sont reportées tableau VI.

\section{Alcaloïdes synthétiques et naturels anticholiner- giques}

L'analyse toxicologique de ces composés est extrêmement délicate en raison des faibles concentrations susceptibles d'exercer une action pharmacologique. Pour l'atropine, dont la demi-vie est de 2 à 5 heures, le $\mathrm{Vd}$ de 1 à $6 \mathrm{l} / \mathrm{kg}$, les concentrations thérapeutiques de 2 à $25 \mathrm{ng} / \mathrm{ml}$ et les concentrations toxiques de 20 à 30 $\mathrm{ng} / \mathrm{ml}(16,36)$, la CG/SM après dérivation puis acquisition en mode sélection d'ions (53), la chromatographie liquide avec paire d'ions (54), ou l'électrophorèse capillaire (55), présentent des limites de quantification respectives de $10 \mathrm{ng} / \mathrm{ml}, 400 \mathrm{ng} / \mathrm{ml}$ et $200 \mathrm{ng} / \mathrm{ml}$ et offrent des performances analytiques pas toujours suffisantes. La technique par CL/SM proposée par Gaillard (22) en revanche répond bien aux critères de sensibilité (tableau III) avec une limite de détection pour l'atropine de $0,09 \mathrm{ng} / \mathrm{ml}$. En ce qui concerne la scopolamine, dont la demi-vie est de 3 à 8 heures, le $\mathrm{Vd}$ de $1,4 \mathrm{l} / \mathrm{kg}$, les concentrations thérapeutiques sont comprises entre 0,1 et $1 \mathrm{ng} / \mathrm{ml}$ (16), cette même technique répond bien aux exigences de la toxicologie hospitalière, avec une lìmite de détection de $0,31 \mathrm{ng} / \mathrm{ml}$ (tableau III). Pour cette molécule, il est possible d'utiliser la $\mathrm{CG} / \mathrm{SM}$ après dérivation puis acquisition en mode sélection d'ions (56), la limite de quantification est de $2 \mathrm{ng} / \mathrm{ml}$. Pour l'hyoscyamine en revanche qui présente une demi-vie de 3 à 8 heures et un Vd de 1,41 , les concentrations thérapeutiques de 0,1 à $1 \mathrm{ng} / \mathrm{ml}$ sont beaucoup trop faibles pour être détectées par cette technique et il faut faire appel à la chromatographie liquide couplée à une double spectrométrie de masse ou $\mathrm{CL} / \mathrm{SM} / \mathrm{SM}$ (57). En ce qui concerne la confirmation d'une intoxication par les morelles, la mise en éviden- 
Tableau VI : Principales caractéristiques pharmacocinétiques des antipsychotiques neuroleptiques phénothiaziniques anticholinergiques selon Wennig (16), Meyer (38), Uges (36).

\begin{tabular}{|l|c|c|c|c|}
\hline \multirow{2}{*}{\multicolumn{1}{c|}{ Principe actif }} & Demi-vie & Vd & \multicolumn{2}{c|}{$\begin{array}{c}\text { Concentrations plasmatiques } \\
\text { (ng/ml ou } \mathbf{\mu g} / \text { l) }\end{array}$} \\
\cline { 4 - 5 } & d'élimination (h) & $\mathbf{( 1 / k g )}$ & Thérapeutiques & Toxiques \\
\hline Chlorpromazine & 30 & 7 & $50-300$ & $500-1000$ \\
\hline Cyamémazine & - & - & $50-400$ & - \\
\hline Fluphénazine & $3-45$ & - & $5-25$ & $50-100$ \\
\hline Lévomépromazine & $15-30$ & $23-42$ & $30-150$ & 500 \\
\hline Perphénazine & $8-12$ & $20-25$ & $1-20$ & $50-100$ \\
\hline Pipotiazine & $8-11$ & - & $1-60$ & 100 \\
\hline Propériciazine & - & - & $5-50$ & 100 \\
\hline Thiopropérazine & - & - & $1-20$ & 100 \\
\hline Thioridazine & $16-30$ & $10-18$ & $200-1000$ & $>1000$ \\
(Mesoridazine $:$ métabolite) & $2-9$ & $3-6$ & $100-1500$ & 3 à 7000 \\
\hline Trifluopérazine & $7-18$ & 160 & $5-50$ & $100-200$ \\
\hline
\end{tabular}

ce de la solanidine peut être réalisée par CG/SM après triple extraction dans les végétaux ou les échantillons biologiques (58).

\section{Conclusion}

Au cours des dix dernières années, des progrès déterminants ont été réalisés en ce qui concerne la réponse analytique aux syndromes cholinergiques et anticholinergiques. Les techniques chromatographiques se sont substituées aux réactions colorimétriques peu précises et surtout peu spécifiques. Le développement de la spectrométrie de masse a largement complété voire supplanté les méthodes immunologiques. La baisse importante des coûts en terme d'investissement a aussi contribué à une plus large implantation de ces équipements dans les laboratoires de toxicologie. Grâce aux progrès fulgurants dans les techniques de couplage chromatographiques ; à l'amélioration des limites de détection ; au progrès dans le domaine informatique pour le traitement des signaux ; mais aussi dans la préparation de l'échantillon ; aujourd'hui parmi les substances cholinergiques et. anticholinergiques, seule la muscarine échappe à l'analyse toxicologique. Cependant, l'arbre ne doit pas cacher la forêt, l'interprétation des données, pour une meilleure connaissance des mécanismes de toxicité et une meilleure prise en charge des malades rend indispensables la confrontation des données analytiques à celles de la clinique. Cette situation nouvelle impose plus que jamais une étroite collaboration entre clinicien et toxicologue analyste.

\section{Références}

1. Schrader G. Die entwicklung neuer insektizide auf grundlage von organischem fluor-und phosphorverbindungen. Verlag Chemie, Weinheim, 1952. Monographie No. 62.

2. Lambert H., Zitoli J.L., Pierrot M, Manel J. Intoxications par les champignons : syndromes mineurs. Encycl Med Chir (Elsevier, Paris), Toxicologie-Pathologie professionnelle, 16-077-B-10, 2000, 10 p.

3. Maroni M., Colosio C., Ferioli A., Fait A. Biological monitoring of pesticide exposure : a review. Organophosphorous pesticides. Toxicology, $2000 ; 143$ : 9-37.

4. Bismuth Ch., Barriot P. Armes chimiques. Dangerosité. Modalités et prise en charge. EMC, 1993 ; 16-650-A-10.

5. Lacassie E., Marquet P., Gaulier J.M., Dreyfuss M.F., Lachâtre G. Exploration analytique des intoxications par les pesticides. Annales de Toxicologie Analytique, 2000 ; $12: 315-24$.

6. Lacassie E., Marquet P., Gaulier J.M., Dreyfuss M.F., Lachâtre G. Sensitive and specific multiresidue methods for the determination of pesticides of various classes in clinical and forensic toxicology. Forensic Sci Int. 2000 ; special issue (accepté pour publication).

7. Singh A.K., Zeleznikar R.J., Drewes L.R. Analysis of soman and sarin in blood utilizing a sensitive gas chromatography-mass spectrometry method. J. Chromatogr. $1985 ; 324: 163-72$.

8. Goransson-Nyberg A., Fredriksson S.A., Karlsson B., Lundstrom M., Cassel G. Toxicokinetics of soman in cerebrospinal fluid and blood of anaesthetized pigs. Arch Toxicol. $1998 ; 72: 459-67$. 
9. Shih M.L., Smith J.R., McMonagle J.D., Dolzine T.W., Gresham V.C. Detection of metabolites of toxic alkylmethylphosphonates in biological samples. Biol Mass Spectrom. $1991 ; 20: 717-23$.

10. Miki A., Katagi M., Tsuchihashi H., Yamashita M. Determination of alkylmethylphosphonic acids, the main metabolites of organophosphorus nerve agents, in biofluids by gas chromatography-mass spectrometry and liquid-liquid-solid-phase-transfer-catalyzed pentafluorobenzylation. J Anal Toxicol. 1999 ; 23 : 86-93.

11. Matsuda Y., Nagao M., Takatori T., Niijima H., Nakajima M. Iwase H., Kobayashi M., Iwadate K. Detection of the sarin hydrolysis product in formalin-fixed brain tissues of victims of the Tokyo subway terrorist attak. Toxicol Appl Pharmacol. $1998 ; 150: 310-20$.

12. Loke W.K., Karlsson B., Waara L., Nyberg A.G., Cassel G.E. Enzyme-based microassay for accurate determination of soman in blood samples. Anal Biochem. 1998 ; $257: 12-9$.

13. Erhard M.H., Kuhlmann R., Szinicz L., Losch U. Detection of the organophosphorus nerve agent soman by an ELISA using monoclonal antibodies. Arch Toxicol. $1990 ; 64: 580-5$.

14. D'Agostino P.A., Hancock J.R., Provost L.R. Packed capillary liquid chromatography-electrospray mass spectrometry analysis of organophosphorus chemical warfare agents. J Chromatogr A. 1999 ; 840 : 289-94.

15. Yamamoto K., Kohda Y., Saitoh Y., Nakagawa F. Determination of ambenonium in biological samples by reversed-phase ion-pair liquid chromatography. J Chromatogr. 1988 ; 433 : 167-76.

16. Wennig R. Laboratory diagnosis of poisonings. Human Toxicology. Descotes J. Elsevier Ed. 1996 : 25-236.

17. Tanzer J.M., Kramer P.A., Schulman P., Willard A.K. A pharmacokinetic and pharmacodynamic study of intravenous pilocarpine in humans. J Dent Res. 1995 ; 74 : 1845-9.

18. Weaver M.L., Tanzer J.M., Kramer P.A. High-performance liquid chromatographic determination of pilocarpine in plasma. J Chromatogr. 1992 ; 581 : 293-6.

19. Birk K.L., Dru J.D., Hsieh J.Y., Demetriades J.L., Matuszewski B.K., Bayne W.F., Woolf E.J., Cairns A.M., Rogers J.D., Musson D.G. Determination of pilocarpic acid in human plasma by capillary gas chromatography with mass-selective detection. J Chromatogr B Biomed Sci Appl. 1998 ; 719 : 93-102.

20. Van de Merbel N.C., Tinke A.P., Oosterhuis B., Jonkman J.H., Bohle J.F. Determination of pilocarpine, isopilocarpine, pilocarpic acid and isopilocarpic acid in human plasma and urine by high-performance liquid chromatography with tandem mass spectrometric detection. J Chromatogr B Biomed Sci Appl. 1998 ; 708 : 103-12.

21. Stijve $T$. High performance thin layor chromatographic determination of the toxic principale of some poisonous mushrooms. Mitt Geb Lebenmittehunters Hyg. 1981 ; 72:44-54.

22. Gaillard Y., Pepin G. Poisoning by plant material : review of human cases and analytical determination of main toxins by high-performance liquid chromatography-(tandem) mass spectrometry. J Chromatogr B. $1999 ; 733: 181-229$.
23. www.vidalpro.net

24. Thomas Y.K. Chan. Anticholinergic poisoning due to chinese herbal medicines. Vet Human Toxicol. 1995 ; $37: 156-7$.

25. Goullé J.P., Bouige D., Berreville E., Rigaud J.P.; Nouveau J. Dépistage toxicologique en médecine hospitalière d'urgence - 2834 bilans. Toxicorama. $1994 ; 6$ : 45-9.

26. Power B.M., Hackett L.P., Dusci L.J., Ilet K.L. Antidepressant toxicity and the need for identification and concentration monitoring in overdose. Clin Pharmacokinet. $1995 ; 29: 154-71$.

27. Goullé J.P., Lacroix C., Nouveau J., Rigaud J.P. Intoxication par les antidépresseurs tricycliques, apport du laboratoire dans la prise en charge médicale. Toxicorama. $1996 ; 8: 21-5$.

28. Goullé J.P., Rigaud J.P., Lacroix C., Nouveau J. Intoxications graves par antidépresseurs tricycliques. A propos de 38 observations. Toxicorama. $1998 ; 10: 255$ 67.

29. Lacassie E., Ragot S., Rabatel J.F., Gaulier J.M., Marquet P., Lachâtre G. Recherche et dosage de 24 antidépresseurs et métabolites dans le sérum par couplage CG/SM. Toxicorama. $1998 ; 10$ : 234-44.

30. Lacassie E., Ragot S., Gaulier J.M., Marquet P., Lachâtre G. Méthode de dosage spécifique de 24 antidépresseurs par couplage chromatographie en phase gazeuse-spectrométrie de masse (CG/SM). Acta Clinica Belgica, 1999 ; suppl. $1: 20-4$.

31. Charlier C., Ansseau M., Gougnard T., Andrien F., Plomteux G. Le monitoring thérapeutique des antidépresseurs. Rev Med Liège. 1997 ; 52 : 336-44.

32. Guitton J., David O., Mialon A., Manchon M. Evaluation d'un automate de chromatographie liquide permettant un screening toxicologique dans les liquides biologiques. Ann Biol Clin. 1993 ; 51 : 611-7.

33. Vincent F., Chartier A., Eysseric H., Marka C., Danel V., Bessard G. Evaluation d'un automate de chromatographie liquide en toxicologie d'urgence. Toxicorama. $1994 ; 6: 7-18$.

34. Sadeg N., François G., Petit B., Dutertre-Catella, Dumontet $\mathrm{M}$. Automated liquid-chromatographic analyzer used for toxicology screening in a general hospital : 12 months' experience. Clin Chem. 1997 ; 43 : 498504.

35. Pechard A., Besson A.S., Mialon A., Berny C., Manchon M. Analyse critique des différentes méthodes utilisées pour le dépistage toxicologique dans un laboratoire d'urgence. Ann Biol Clin. 1999 ; 57 : 525-37.

36. Uges DRA. Therapeutic and toxic drug concentration. TIAFT Bulletin. 1996 ; 26 (1 suppl.) : 34 pp.

37. Rosario-Repetto M., Repetto M. Therapeutic, toxic, and lethal concentrations of 73 drugs affecting respiratory system in human fluids. Clin Toxicol. $1998 ; 36: 287$ 93.

38. Meyer F.P. Indicative therapeutic and toxic drug concentrations in plasma : a tabulation. International Journal of Clinical Pharmacology and Therapeutics. $1994 ; 32: 71$ 81. 
39. Allender W.J., Archer A.W. Liquid chromatographic analysis of promethazine and its major metabolites in human postmortem material. J Forensic Sci. $1984 ; 29$ : 515-26.

40. Leelavathi D.E., Dressler D.E., Soffer E.F., Yachetti S.D., Knowles J.A. Determination of promethazine in human plasma by automated high-performance liquid chromatography with electrochemical detection and by gas chromatography-mass spectrometry. J Chromatogr. 1985 ; 339 : 105-15.

41. Bagli M., Rao M.L., Hoflich G. Quantification of chlorprothixene, levomepromazine and promethazine in human serum using high-performance liquid chromatography with coulometric electrochemical detection. J Chromatogr B Biomed Appl. 1994 ; 657 : 141-48.

42. Liu J., Stewart J.T. Quantitation of promethazine enantiomers in human serum using a chiralcel OJ-R column and mixed-mode disc solid-phase extraction. J Pharm Biomed Anal. 1997 ; 16 : 303-9.

43. Miyamoto Y. Highly sensitive determination of chlorpheniramine as fluorescence derivative by high-performance liquid chromatography. J Chromatogr. 1987 ; $420: 63-72$.

44. Yamaguchi M., Monji H., Yamashita K., Aoki I., Yashiki T. Sensitive high-performance liquid chromatographic determination of chlorpheniramine in human serum using column switching. J Chromatogr B Biomed Appl. $1994 ; 661: 168-72$.

45. Ponder G.W., Stewart J.T. A liquid chromatographic method for the determination of promethazine enantiomers in human urine and serum using solid-phase extraction and fluorescence detection. J Pharm Biomed Anal. $1995 ; 13: 1161-66$.

46. Kumar S., Rurak D.W., Riggs K.W. Simultaneous determination of diphenhydramine, its $\mathrm{N}$-oxide metabolite and their deuterium-labeled analogues in ovine plasma and urine using liquid chromatography/electrospray tandem mass spectrometry. J Mass Spectrom. 1998 ; 33 : 1171-81.

47. Celma C., Allue J.A., Prunonosa J., Peraire C., Obach R. Simultaneous determination of paracetamol and chlorpheniramine in human plasma by liquid chromatography-tandem mass spectrometry. J Chromatogr A. 2000 ; $870: 77-86$
48. Desage M., Rousseau-Tsangaris M., Lecompte D., Brazier J.L. Quantitation of trihexyphenidyl from plasma using a mass-selective detector and electron-impact ionization. J Chromatogr. 1991 ; 571 : 250-6.

49. Vargas G., Havel J., Babackova L., Patocka J. Determination of drugs used as anti-Parkinson's disease drugs in urine and serum by capillary electrophoresis. $\mathbf{J}$ Capillary Electrophor. $1998 ; 5: 153-58$.

50. Capka V., Xu Y., Chen Y.H. Stereoselective determination of trihexyphenidyl in human serum by LC-ESI-MS. J Pharma Biomed Anal. 1999 ; 21 : 507-17.

51. Bun H., Monjanel-Mouterde S., Audebert C., Vincent Du Laurier M., Durand A., Cano J.P. Pharmacokinetics of tropatepine in healthy volunteers. Clin Drug Invest. 1997 ; 14 : 53-60.

52. Rosario-Repetto M., Repetto $M$. Concentrations in human fluids : 101 drugs affecting the digestive system and metabolism. Clin Toxicol. 1999 ; $37: 1-8$.

53. Saady J.J., Poklis A. Determination of atropine in blood by gas chromatography/mass spectrometry. J Anal Toxicol. $1989 ; 13: 296-9$.

54. Buch U., Isenberg E., Buch H.P. HPLC assay for atropine in serum and protein solutions after in vitro addition of the tropane alkaloid. Methods Find Exp Clin Pharmacol. $1994 ; 16: 361-5$.

55. Plaut O., Staub C. Determination of atropine in biological fluids by micellar electrokinetic capillary chromatography in the presence of strychnine and tetracaine. Electrophoresis. $1998 ; 19$ : 3003-7.

56. Deutsch J., Soncrant T.T., Greig N.H., Rapoport S.I. Electron-impact ionization detection of scopolamine by gas chromatography-mass spectrometry in rat plasma and brain. J Chromatogr. 1990 ; $528: 325-31$.

57. Xu A., Havel J., Linderholm K., Hulse J. Development and validation of an LC/MS/MS method for the determination of L-hyoscyamine in human plasma. J Pharm Biomed Anal. 1995 ; 14 : 33-42.

58. Hostege D.M., Seiber J.N., Galey F.D. Rapid multiresidue screen for alcaloids in plant material and biological samples. J Agric Food Chem. 1995 ; 43 : 691. 